\title{
ON SOME COMPOSITION FORMULAS
}

\section{J. HORVÁTH}

1. We shall denote by $x=\left(x_{1}, \cdots, x_{n}\right)$ and $\xi=\left(\xi_{1}, \cdots, \xi_{n}\right)$ the points of the $n$-dimensional real Euclidean space $R^{n}$. We put $r=|x|$ $=\left(x_{1}^{2}+\cdots+x_{n}^{2}\right)^{1 / 2}$ and similarly $\rho=|\xi|$.

The well known kernels of Marcel Riesz [9, p. 16; 10, pp. 45-48] are defined by

$$
R_{\alpha}=\frac{\Gamma\left(\frac{n-\alpha}{2}\right)}{\pi^{n / 2} 2^{\alpha} \Gamma(\alpha / 2)} \cdot \frac{1}{r^{n-\alpha}},
$$

except for the singular values $\alpha=-2 \nu$ and $\alpha=n+2 \nu(\nu=0,1,2, \cdots)$. By analytic continuation one is led to define $R_{-2 \nu}=(-\Delta)^{\nu} \delta$, where $\Delta$ is the Laplace operator and $\delta$ Dirac's delta. For the other exceptional values we have

$$
R_{n+2 \nu}=\frac{2(-1)^{\nu}}{\pi^{n / 2} 2^{n+2 \nu} \Gamma(n / 2+\nu) \nu !} r^{2 \nu}\left(\log \frac{1}{\pi r}+A_{n, \nu}\right),
$$

where

$$
A_{n, \nu}=\frac{1}{2}\left(1+\frac{1}{2}+\cdots+\frac{1}{\nu}-C\right)+\frac{1}{2} \frac{\Gamma^{\prime}(n / 2+\nu)}{\Gamma(n / 2+\nu)}
$$

and $C$ is Euler's constant [11, Formula (VII, 7; 14), p. 114; 9, p. 22]. With these definitions the Riesz kernel satisfies the composition formula

$$
R_{\alpha} * R_{\beta}=R_{\alpha+\beta},
$$

which follows, for instance, from the fact that the Fourier transform of $R_{\alpha}$ is

$$
\mathcal{F}\left(R_{\alpha}\right)=\frac{1}{(2 \pi)^{\alpha}} \cdot \frac{1}{\rho^{\alpha}}
$$

$[5$, p. $151 ; 11$, pp. 113-114].

2. Let $\nabla$ be the nabla operator defined by

$$
\nabla=\sum_{i=1}^{n} e_{i} \frac{\partial}{\partial x_{i}}
$$

Received by the editors September 24, 1958. 
where $e_{1}, \cdots, e_{n}$ is the canonical basis of $R^{n}$. Let us put

$$
N_{\alpha}=-\nabla R_{\alpha+1} \text {. }
$$

$N_{\alpha}$ is a vector valued kernel and is given for $\alpha \neq-1-2 \nu, \alpha \neq n+2 \nu+1$ $(\nu=0,1,2, \cdots)$ by

$$
N_{\alpha}=\frac{\Gamma\left(\frac{n-\alpha+1}{2}\right)}{\pi^{n / 2} 2^{\alpha} \Gamma\left(\frac{\alpha+1}{2}\right)} \frac{x}{r^{n-\alpha+1}} .
$$

For $\alpha \neq n-1$ this follows taking the gradient of (1), while for $\alpha=n-1$ we must take the gradient of (2) with $\nu=0$ and use the relation

$$
\nabla \log \frac{1}{\pi r}=-\frac{x}{r^{2}}
$$

The kernels (6) satisfy the composition formula

$$
N_{\alpha} * N_{\beta}=-R_{\alpha+\beta},
$$

where the convolution of the two vector valued distributions must be taken in the sense of scalar products $[10$, p. 30]. Indeed we have $N_{\alpha} * N_{\beta}=\nabla R_{\alpha+1} * \nabla R_{\beta+1}=\Delta R_{\alpha+\beta+2}=-R_{-2} * R_{\alpha+\beta+2}=-R_{\alpha+\beta}$. Alternately (9) can be deduced from

$$
\mathfrak{F}\left(N_{\alpha}\right)=-\frac{i}{(2 \pi)^{\alpha}} \frac{\xi}{\rho^{\alpha+1}} .
$$

Formula (9) contains several important and well-known particular cases:

(a) $\alpha=\beta=0$; then we have the Hilbert-Riesz kernel

$$
N_{0}=\frac{\Gamma\left(\frac{n+1}{2}\right)}{\pi^{(n+1) / 2}} \frac{x}{r^{n+1}}
$$

and (9) yields $N_{0} * N_{0}=-\delta$. This reciprocity formula, due essentially to Marcel Riesz, was proved first in [6] and was used in [2, p. 299] and in [3, Formula (19), p. 907].

(b) $\alpha=1, \beta=-1$; then we have

$$
N_{1}=\frac{\Gamma\left(\frac{n}{2}\right)}{2 \pi^{n / 2}} \frac{x}{r^{n}}, N_{-1}=-\nabla \delta
$$


and (9) yields $\nabla N_{1}=\delta$. Now $N_{1}$ is the kernel of Newtonian force and the result can be expressed saying that forming the gradient and forming the Newtonian force are operations inverse to each other. This result was communicated to me orally by Marcel Riesz and the present note grew out of an attempt to give it a more general setting.

(c) $\alpha=-1, \beta=0$; in this case (9) yields $\Lambda=\nabla \delta * N_{0}=R_{-1}$ or $\Lambda * \Lambda$ $=-\Delta$. This result figures in [3, Formula (24), p. 909].

3. Now we arrive at the main part of the paper. Let $Q=\sum_{j=0}^{\infty} Q_{j}$ be the commutative graded algebra attached to the Laplace operator $\left[7\right.$, p. $56 ; 8$, Example 1]. $Q$ is generated by the elements $e_{1}, \cdots, e_{n}$ belonging to $Q_{1}$ (and by $1 \in Q_{0}$ ), which satisfy the relation $e_{1}^{2}+\cdots$ $+e_{n}^{2}=0 . Q_{0}$ is identified with $R$ and $Q_{1}$ with $R^{n}$. Taking $x=x_{1} e_{1}+\cdots$ $+x_{n} e_{n}$ with indeterminate coefficients $x_{1}, \cdots, x_{n}$, the components of $x^{j}$ with respect to a basis of $Q_{j}$ form a maximal system of homogeneous harmonic polynomials of degree $j$.

With these identifications, $\nabla$ operates on distributions taking their values in $Q$ and $\nabla^{i}(j=0,1,2, \cdots)$ is well defined. Let us write

$$
K_{\alpha, j}=(-\nabla)^{j} R_{\alpha+j} .
$$

For the values $\alpha+j \neq-2 \nu$ and $\alpha-j \neq n+2 \nu(\nu=0,1,2, \cdots) K_{\alpha, j}$ is given by the formula

$$
K_{\alpha, j}=\frac{\Gamma\left(\frac{n-\alpha+j}{2}\right)}{\pi^{n / 2} 2^{\alpha} \Gamma\left(\frac{\alpha+j}{2}\right)} \frac{x^{j}}{r^{n-\alpha+j}} .
$$

For the values $\alpha+j \neq n+2 \nu(\nu=0,1, \cdots, j-1)$ this follows taking $j$ times the gradient of (1) and using the relation $\nabla x=e_{1}^{2}+\cdots+e_{n}^{2}=0$. To calculate $(-\nabla)^{i} R_{n+2 \nu}$ with $\alpha+j=n+2 \nu(\nu=0,1, \cdots, j-1)$ formula (2) must be taken in account. First, an induction [7, pp. 61-62] which uses (8) and $\nabla x=0$ shows that for $1 \leqq \mu \leqq \nu$

$$
\begin{aligned}
& \nabla^{\mu} r^{2 \nu}\left(\log \frac{1}{\pi r}+A_{n, \nu}\right) \\
& \quad=2 \nu(2 \nu-2) \cdots(2 \nu-2 \mu+2) x^{\mu} r^{2 \nu-2 \mu}\left(\log \frac{1}{\pi r}+A_{n, \nu}\right)+\alpha_{\mu} x^{\mu} r^{2 \nu-2 \mu},
\end{aligned}
$$

holds, where $\alpha_{\mu}$ is a constant. In particular we have

$$
\nabla^{\nu} r^{2 \nu}\left(\log \frac{1}{\pi r}+A_{n, \nu}\right)=2^{\nu} \nu ! x^{\nu}\left(\log \frac{1}{\pi r}+A_{n, \nu}\right)+\alpha_{\nu} x^{\nu}
$$


From here it follows, using again (8) and $\nabla x=0$, that

$$
\nabla^{\nu+1} r^{2 \nu}\left(\log \frac{1}{\pi r}+A_{n, \nu}\right)=-2^{\nu} \cdot \nu ! \frac{x^{\nu+1}}{r^{2}} .
$$

Since obviously

$$
\nabla^{j-\nu-1} \frac{x^{\nu+1}}{r^{2}}=(-1)^{j-\nu-1} 2^{j-\nu-1}(j-\nu-1) ! \frac{x^{j}}{r^{2 j-2 \nu}},
$$

the expression of $K_{\alpha, j}$ follows from (2), (12), (13).

The $K_{\alpha, j}$ satisfy the composition formula

$$
K_{\alpha, j} * K_{\beta, l}=K_{\alpha+\beta, j+l},
$$

where the convolution must be taken in the sense of the multiplication in $Q$. This can be seen either directly from the definition (11) using (3) or from the formula

$$
\mathcal{F}\left(K_{\alpha, j}\right)=(-2 \pi i \xi)^{i} F\left(R_{\alpha+j}\right)=\frac{(-i)^{j}}{(2 \pi)^{\alpha}} \frac{\xi^{j}}{\rho^{\alpha+j}} .
$$

4. Final remarks. (a) For $\alpha=0$ we obtain the Hilbert kernels $H_{j}=K_{0, j}$ given by

$$
H_{0}=\delta, \quad H_{j}=\frac{\Gamma\left(\frac{n+j}{2}\right)}{\pi^{n / 2} \Gamma\left(\frac{j}{2}\right)} \frac{x^{j}}{r^{n+j}} \quad(j=1,2,3, \cdots)
$$

and introduced in $\left[7\right.$, p. 59]. (14) yields the formula $H_{j} * H_{l}=H_{j+l}$ proved in [7].

(b) Let $Y_{j}(x)$ be a homogeneous harmonic polynomial of degree $j$. Considering components, we obtain from (15) that the Fourier transform of

$$
\frac{Y_{j}(x)}{r^{n-\alpha+j}}
$$

is

$$
\frac{(-i)^{j} \pi^{n / 2-\alpha} \Gamma\left(\frac{\alpha+\jmath}{2}\right)}{\Gamma\left(\frac{n-\alpha+\jmath}{2}\right)} \frac{Y_{j}(\xi)}{\rho^{\alpha+i}}
$$


for $\alpha+j \neq-2 \nu, \alpha-j \neq n+2 \nu(\nu=0,1,2, \cdots)$. This result has been obtained by Bochner [1, Formula (7), p. 807] in a quite different way. The absence of the factor $(2 \pi)^{\alpha-n / 2}$ is due to the different normalization of the Fourier transform.

(c) The expression (16) is of the form

$$
k(\sigma) / r^{n-\alpha}
$$

where $k(\sigma)$ is a continuous function defined on the unit sphere. If $0 \leqq \alpha<n$, then the restriction of (17) to $|x| \geqq 1$ belongs to $L^{\cdot}$ for any $s>n /(n-\alpha)$ and thus (17) defines a distribution $K \in D_{L^{\circ}}^{\prime}$ [11, pp. 55-61]. Thus $T \rightarrow K * T$ is a continuous map from $\mathscr{D}_{L^{p}}^{\prime}$ into $\mathscr{D}_{L^{q}}^{\prime}$ with $1 / q=1 / s+1 / p-1<1 / p-\alpha / n \quad(1<p<q<\infty)$. Actually it can be shown (using for instance the convexity theorem of Marcinkiewicz [12]) that $f \rightarrow K * f$ is a continuous map from $L^{p}$ into $L^{q}$ and $T \rightarrow K * T$ is a continuous map ${ }^{1}$ from $D_{L^{p}}^{\prime}$ into $D_{L^{q}}^{\prime}$, with $1 / q=1 / p-\alpha / n$. For $k(\sigma) \equiv 1$ this is Sobolev's theorem [11, p. 37], for $\alpha=0$ that of Calderón and Zygmund [2]. It seems from the brief indications given in [4] that Cotlar is preparing a paper on these questions.

\section{REFERENCES}

1. S. Bochner, Theta relations with spherical harmonics, Proc. Nat. Acad. Sci. U.S.A. vol. 37 (1951) pp. 804-808.

2. A. P. Calderon and A. Zygmund, On singular integrals, Amer. J. Math. vol. 78 (1956) pp. 289-309.

3. - Singular integral operators and differential equations, Amer. J. Math. vol. 79 (1957) pp. 901-921.

4. M. Cotlar, Generalized potential operators, Abstracts of short communications, International Congress of Mathematicians, Edinburgh, 1958, pp. 78-79.

5. J. Deny, Les potentiels d'énergie finie, Acta Math. vol. 82 (1950) pp. 107-183.

6. J. Horváth, Sur les fonctions conjuguées a plusieurs variables, Nederl. Akad. Wetensch. Indag. Math. vol. 15 (1953) pp. 17-29.

7. - Singular integral operators and spherical harmonics, Trans. Amer. Math. Soc. vol. 82 (1956) pp. 52-63.

8. - Basic sets of polynomial solutions for partial differential equations, Proc. Amer. Math. Soc. vol. 9 (1958) pp. 569-575.

9. M. Riesz, L'intégrale de Riemann-Liouville et le problème de Cauchy, Acta Math. vol. 81 (1949) pp. 1-223.

10. L. Schwartz, Théorie des distributions, vol. 1, Paris, 1950.

11. — ibid., vol. 2, Paris, 1951.

12. A. Zygmund, On a theorem of Marcinkiewicz concerning interpolation of operations, J. Math. Pures Appl. vol. 35 (1956) pp. 223-248.

\section{UNIVERSITY OF MARYLAND}

${ }^{1}$ The corollary to the closed graph theorem has been stated on p. 55 of [7] for complete metrizable spaces, but it holds, like the closed graph theorem itself, also for $(\mathscr{L F})$ spaces and can therefore be applied to the spaces $D_{L^{\prime}}^{\prime}$. 